\title{
Optimizing care for geriatric cancer patients: the greatest challenge in medical oncology?
}

Rupert Bartsch (iD)

Received: 15 October 2019 / Accepted: 15 October 2019

(c) Springer-Verlag GmbH Austria, part of Springer Nature 2019

Fundamental progress has been achieved in the treatment of solid cancers over the last two decades and patients' long-term outcome has improved accordingly. With monoclonal antibodies, small-molecule tyrosine kinase inhibitors and more recently, immune checkpoint modulators, a plethora of highly active novel drugs has become available. But are truly all patients benefitting from these successes? In an elderly population of breast cancer patients, the HER2positive subtype was still associated with the worst prognosis as shown in a recent publication [1] despite the excellent survival results achieved within clinical trials [2].

Indeed, optimization of the treatment of elderly patients may be the greatest challenge medical oncologists are facing today. Comorbid conditions, polypharmacy, decreased functional reserve, and age-related changes in pharmacokinetics result in impaired tolerability of anticancer drugs. Underrepresentation in clinical trials adds to this problem while, on the other hand, demographic changes with a growing older population results in an ever-increasing number of elderly cancer patients [3].

In their study published in this issue of MEMO, Balic et al. analyzed the prevalence of comorbid conditions in more than a thousand patients with solid cancer in Austria treated in 2011 [4]. With a median age of 64 years, the population represents clinical reality and clinically relevant comorbidities were present in $86 \%$ of patients. Not surprisingly, there was a significant association between the number of comorbid conditions and age ( $\chi^{2}$ test; $\left.p<0.001\right)$ and the vast ma- jority of elderly patients (89\%) presented with more than three comorbidities, emphasizing the huge degree of this problem. As this study was not intended as a longitudinal observation, the influence of age and comorbidities on overall survival was not assessable but a relationship between comorbidities and longterm outcome in oncology is already well established [5].

Clearly, the authors need to be congratulated to their effort of again bringing the problem of geriatric oncology into focus. But where should we move from here? As pointed out by the authors, young oncologists should receive comprehensive training in the management of the most relevant noncancerous diseases seen in an elderly population. A major step forward in the care of geriatric cancer patients in Austria, however, would require close cooperation of medical oncologists with geriatricians and implementation of a comprehensive geriatric assessment (CGA) in the clinical routine setting which was shown to improve compliance, treatment tolerability, quality of life, and survival in an elderly population and is therefore the only basis for optimal age- and risk-adapted decision making [6]. Finally, the urgent need for clinical trials specifically designed for the needs of geriatric cancer patients must be emphasized and so far, a lack of local public funding has hampered the participation of Austrian centers in international studies

Conflict of interest R. Bartsch declares that he has no competing interests.

\footnotetext{
R. Bartsch ( $₫)$

Department of Medicine 1, Division of Oncology, and Comprehensive Cancer Center, Medical University of Vienna, Währinger Gürtel 18-20, 1090 Vienna, Austria rupert.bartsch@meduniwien.ac.at
} 


\section{References}

1. Bergen ES, Tichy C, Berghoff AS, et al. Prognostic impact of breast cancer subtypes in elderly patients. Breast Cancer Res Treat. 2016;157(1):91-9.

2. Swain SM, KimSB, Cortés J, et al. Pertuzumab, trastuzumab, and docetaxel for HER2-positive metastatic breast cancer (CLEOPATRA study): overall survival results from a randomised, double-blind, placebo-controlled, phase 3 study. Lancet Oncol. 2013;14(6):461-71.

3. Zeber JE, Copeland LA, Hosek BJ, Karnad AB, Lawrence VA, Sanchez-Reilly SE. Cancer rates, medical comorbidities, and treatment modalities in the oldest patients. Crit Rev OncolHematol. 2008;67(3):237-342.

4. Balic M, Hilbe W, Gusel S, et al. Prevalence of comorbidity in cancer patients scheduled for systemic anticancer treatment in Austria. A cross-sectional multicenter observational study. memo. 2019;12(4). https://doi.org/10. 1007/s12254-019-00542-7.
5. Yancik R, Wesley MN, Ries LA, Havlik RJ, Edwards BK, Yates JW. Effect of age and comorbidity in postmenopausal breast cancer patients aged 55 years and older. JAMA. 2001;285(7):885-92.

6. Pallis AG, Fortpied C, Wedding U, et al. EORTC elderly task force position paper: approach to the older cancer patient. Eur J Cancer. 2010;46:1502-13.

Publisher's Note Springer Nature remains neutral with regard to jurisdictional claims in published maps and institutional affiliations.

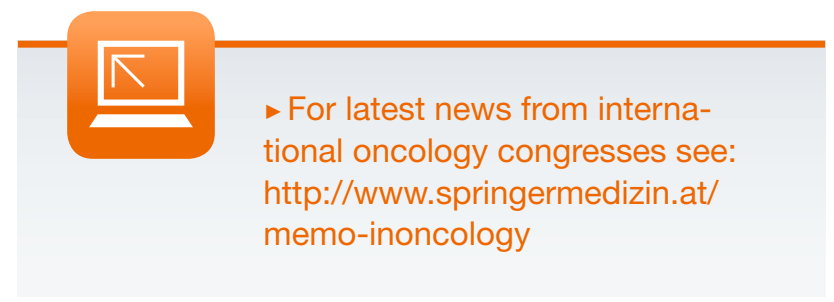

